\title{
Strong convergent result for quasi-nonexpansive mappings in Hilbert spaces
}

Ming $\operatorname{Tian}^{*}$ and Xin Jin

* Correspondence: tianming1963@126.com College of Science, Civil Aviation University of China, Tianjin 300300, China

\section{Abstract}

In this article, we study an iterative method over the class of quasi-nonexpansive mappings which are more general than nonexpansive mappings in Hilbert spaces. Our strong convergent theorems include several corresponding authors' results.

2000 MSC: 58E35; 47H09; 65J15.

Keywords: quasi-nonexpansive mapping, Lipschitzian continuous, strongly monotone, nonlinear operator, fixed point, viscosity method

\section{Introduction}

Let $H$ be a real Hilbert space with inner product $\langle\cdot, \cdot\rangle$, and induced norm $\|\cdot\|$. A mapping $T: H \rightarrow H$ is called nonexpansive if $\|T x-T y\| \leq\|x-y\|$ for all $x, y \in H$. The set of the fixed points of $T$ is denoted by $\operatorname{Fix}(T):=\{x \in H: T x=x\}$.

The viscosity approximation method was first introduced by Moudafi [1] in 2000. Starting with an arbitrary initial $x_{0} \in H$, define a sequence $\left\{x_{n}\right\}$ generated by

$$
x_{n+1}=\frac{\varepsilon_{n}}{1+\varepsilon_{n}} f\left(x_{n}\right)+\frac{1}{1+\varepsilon_{n}} T x_{n}, \quad \forall n \geq 0,
$$

where $f$ is a contraction with a coefficient $\alpha \in[0,1)$ on $H$, i.e., $\|f(x)-f(y)\| \leq \alpha|| x$ $y \|$ for all $x, y \in H, T$ is nonexpansive, and $\left\{\varepsilon_{n}\right\}$ is a sequence in $(0,1)$ satisfying the following given conditions:
(i1) $\lim _{n \rightarrow \infty} \varepsilon_{n}=0$;
(i2) $\sum_{n=0}^{\infty} \varepsilon_{n}=\infty$;
(i3) $\lim _{n \rightarrow \infty}\left(\frac{1}{\varepsilon_{n}}-\frac{1}{\varepsilon_{n+1}}\right)=0$.

It is proved that the sequence $\left\{x_{n}\right\}$ generated by (1.1) converges strongly to the unique solution $x^{*} \in C(C:=\operatorname{Fix}(T))$ of the variational inequality:

$$
\left\langle(I-f) x^{*}, x-x^{*}\right\rangle \geq 0, \quad \forall x \in \operatorname{Fix}(T) .
$$

In 2003, Xu [2] proved that the sequence $\left\{x_{n}\right\}$ defined by the below process where $T$ is also nonexpansive, started with an arbitrary initial $x_{0} \in H$ :

$$
x_{n+1}=\alpha_{n} b+\left(I-\alpha_{n} A\right) T x_{n}, \quad \forall n \geq 0,
$$


converges strongly to the unique solution of the minimization problem (1.3) when the sequence $\left\{\alpha_{n}\right\}$ satisfies certain conditions:

$$
\min _{x \in C} \frac{1}{2}\langle A x, x\rangle-\langle x, b\rangle
$$

where $C$ is the set of fixed points set of $T$ on $H$ and $b$ is a given point in $H$.

In 2006, Marino and Xu [3] combined the iterative method (1.2) with the viscosity approximation method (1.1) and considered the following general iterative method:

$$
x_{n+1}=\alpha_{n} \gamma f\left(x_{n}\right)+\left(I-\alpha_{n} A\right) T x_{n}, \quad \forall n \geq 0 .
$$

It is proved that if the sequence $\left\{\alpha_{n}\right\}$ satisfies appropriate conditions, the sequence $\left\{x_{n}\right\}$ generated by (1.4) converges strongly to the unique solution of the variational inequality:

$$
\langle(\gamma f-A) \tilde{x}, x-\tilde{x}\rangle \leq 0, \quad \forall x \in C,
$$

or equivalently $\tilde{x}=P_{\operatorname{Fix}(T)}(I-A+\gamma f) \tilde{x}$, where $C$ is the fixed point set of a nonexpansive mapping $T$.

In 2009, Maingè [4] considered the viscosity approximation method (1.1), and expanded the strong convergence to quasi-nonexpansive mappings in Hilbert space.

In 2010, Tian [5] considered the following general iterative method under the frame of nonexpansive mappings:

$$
x_{n+1}=\alpha_{n} \gamma f\left(x_{n}\right)+\left(I-\mu \alpha_{n} F\right) T x_{n}, \quad \forall n \geq 0,
$$

and gave some strong convergent theorems.

Very recently, Tian [6] extended (1.6) to a more general scheme, that is: the mapping $f: H \rightarrow H$ is no longer a contraction but a $L$-Lipschitzian continuous operator with coefficient $L>0$, and proved that if the sequence $\left\{\alpha_{n}\right\}$ satisfies appropriate conditions, the sequence $\left\{x_{n}\right\}$ generated by $x_{n+1}=\alpha_{n} \gamma f\left(x_{n}\right)+\left(I-\mu \alpha_{n} F\right) T x_{n}$ converges strongly to the unique solution $\tilde{x} \in \operatorname{Fix}(T)$ of the variational inequality where $T$ is still nonexpansive:

$$
\langle(\gamma f-\mu F) \tilde{x}, x-\tilde{x}\rangle \leq 0, \quad \forall x \in \operatorname{Fix}(T) .
$$

Motivated by Maingè [4] and Tian [6], we consider the following iterative process:

$$
\left\{\begin{array}{l}
x_{0}=x \in H \quad \text { arbitrarily chosen, } \\
x_{n+1}=\alpha_{n} \gamma f\left(x_{n}\right)+\left(I-\alpha_{n} \mu F\right) T_{\omega} x_{n}, \quad \forall n \geq 0,
\end{array}\right.
$$

where $f$ is $L$-Lipschitzian, $T_{\omega}=(1-\omega) I+\omega T$, and $T$ is a quasi-nonexpansive mapping. Under some appropriate conditions on $\omega$ and $\left\{\alpha_{n}\right\}$, we obtain strong convergence over the class of quasi-nonexpansive mappings in Hilbert spaces. Our result is more general than Maingè's [4] conclusion.

\section{Preliminaries}

Throughout this article, we write $x_{n} \rightarrow x$ to indicate that the sequence $\left\{x_{n}\right\}$ converges weakly to $x . x_{n} \rightarrow x$ implies that the sequence $\left\{x_{n}\right\}$ converges strongly to $x$. The following lemmas are useful for our article.

The following statements are valid in a Hilbert space $H$ : for each $x, y \in H, t \in[0,1]$ 
(i) $\|x+y\| \leq\|x\|^{2}+2\langle y, x+y\rangle$;

(ii) $\|(1-t) x+t y\|^{2}=(1-t)\|x\|^{2}+t\|y\|^{2}-(1-t) t\|x-y\|^{2}$;

(iii) $\langle x, y\rangle=-\frac{1}{2}\|x-y\|^{2}+\frac{1}{2}\|x\|^{2}+\frac{1}{2}\|y\|^{2}$.

Lemma 2.1. Let $f: H \rightarrow H$ be a L-Lipschitzian continuous operator with coefficient $L>$ 0. F: $H \rightarrow H$ is a $\kappa$-Lipschitzian continuous and $\eta$-strongly monotone operator with $\kappa>$ 0 and $\eta>0$. Then, for $0<\gamma \leq \mu \eta / L$,

$$
\left\langle x-\gamma_{,}(\mu F-\gamma f) x-(\mu F-\gamma f) \gamma\right\rangle \geq(\mu \eta-\gamma L)\|x-\gamma\|^{2} .
$$

That is, $\mu F-\gamma f$ is strongly monotone with coefficient $\mu \eta-\gamma L$.

Lemma 2.2. [4] Let $T_{\omega}:=(1-\omega) I+\omega T$, with $T$ quasi-nonexpansive on $H$, Fix $(T) \neq$ $\varnothing$, and $\omega \in(0,1]$. Then, the following statements are reached:

(a1) $\operatorname{Fix}(T)=\operatorname{Fix}\left(T_{\omega}\right)$;

(a2) $T_{\omega}$ is quasi-nonexpansive;

(a3) $\left\|T_{\omega} x-q\right\|^{2} \leq\|x-q\|^{2}-\omega(1-\omega)\|T x-x\|^{2}$ for all $x \in H$ and $q \in$ Fix $(T)$;

(a4) $\left\langle x-T_{\omega} x, x-q\right\rangle \geq \frac{\omega}{2}\|x-T x\|^{2}$ for all $x \in H$ and $q \in$ Fix $(T)$.

Proposition 2.3. From the equality (iii) and the fact that $T$ is quasi-nonexpansive, we have

$$
\langle x-T x, x-q\rangle=-\frac{1}{2}\|T x-q\|^{2}+\frac{1}{2}\|x-T x\|^{2}+\frac{1}{2}\|x-q\|^{2} \geq \frac{1}{2}\|x-T x\|^{2} .
$$

(a4) is easily deduced by $I-T_{\omega}=\omega(I-T)$ and the previous inequality.

Lemma 2.4. [7] Let $\left\{\Gamma_{n}\right\}$ be a sequence of real numbers that does not decrease at infinity, in the sense that there exist a subsequence $\left\{\Gamma_{n_{j}}\right\}_{j_{\geq 0}}\left\{\Gamma_{n}\right\}$ which satisfies $\Gamma_{n_{j}}<\Gamma_{n_{j}+1}$ for all $j \geq 0$. Also, consider the sequence of integers $\{\tau(n)\}_{n \geq n_{0}}$ defined by

$$
\tau(n)=\max \left\{k \leq n \mid \Gamma_{k}<\Gamma_{k+1}\right\} .
$$

Then, $\{\tau(n)\}_{n \geq n_{0}}$ is a nondecreasing sequence verifying $\lim _{n \rightarrow \infty} \tau(n)=\infty$ and for all $n \geq$ $n_{0}$, it holds that $\Gamma_{\tau(n)}<\Gamma_{\tau(n)+1}$ and we have

$$
\Gamma_{n} \leq \Gamma_{\tau(n)+1} .
$$

Recall the metric projection $P_{K}$ from a Hilbert space $H$ to a closed convex subset $K$ of $H$ is defined: for each $x \in H$ the unique element $P_{K} x \in K$ such that

$$
\left\|x-P_{K} x\right\|:=\inf \{\|x-y\|: y \in K\} .
$$

Lemma 2.5. Let $K$ be a closed convex subset of $H$. Given $x \in H$, and $z \in K, z=P_{K} x$, if and only if there holds the inequality:

$$
\langle x-z, y-z\rangle \leq 0, \quad \forall y \in K .
$$

Lemma 2.6. If $x^{*}$ is the solution of the variational inequality (1.7) with $T: H \rightarrow H$ demi-closed and $\left\{y_{n}\right\} \in H$ is a bounded sequence such that $\left\|T y_{n}-y_{n}\right\| \rightarrow 0$, then

$$
\liminf _{n \rightarrow \infty}\left\langle(\mu F-\gamma f) x^{*}, y_{n}-x^{*}\right\rangle \geq 0 .
$$


Proof. We assume that there exists a subsequence $\left\{y_{n_{j}}\right\}$ of $\left\{y_{n}\right\}$ such that $y_{n_{j}} \rightarrow \tilde{\gamma}$. From the given conditions $\left\|T y_{n}-y_{n}\right\| \rightarrow 0$ and $T: H \rightarrow H$ demi-closed, we have that any weak cluster point of $\left\{y_{n}\right\}$ belongs to the fixed point set Fix $(T)$. Hence, we conclude that $\tilde{y} \in \operatorname{Fix}(T)$, and also have that

$$
\liminf _{n \rightarrow \infty}\left\langle(\mu F-\gamma f) x^{*}, y_{n}-x^{*}\right\rangle=\lim _{j \rightarrow \infty}\left\langle(\mu F-\gamma f) x^{*}, y_{n_{j}}-x^{*}\right\rangle .
$$

Recalling (1.7), we immediately obtain

$$
\liminf _{n \rightarrow \infty}\left\langle(\mu F-\gamma f) x^{*}, y_{n}-x^{*}\right\rangle=\left\langle(\mu F-\gamma f) x^{*}, \tilde{y}-x^{*}\right\rangle \geq 0 .
$$

This completes the proof.

\section{Main results}

Let $H$ be a real Hilbert space, let $F$ be a $\kappa$-Lipschitzian and $\eta$-strongly monotone operator on $H$ with $k>0, \eta>0$, and let $T$ be a quasi-nonexpansive mapping on $H$, and $f$ is a $L$-Lipschitzian mapping with coefficient $L>0$ for all $x, y \in H$. Assume the set Fix $(T)$ of fixed points of $T$ is nonempty and we note that $F i x(T)$ is closed and convex.

Theorem 3.1. Let $0<\mu<2 \eta / \kappa^{2}, 0<\gamma<\mu\left(\eta-\frac{\mu \kappa^{2}}{2}\right) / L=\tau / L$, and start with an arbitrary chosen $x_{0} \in H$, let the sequence $\left\{x_{n}\right\}$ be generated by

$$
x_{n+1}=\alpha_{n} \gamma f\left(x_{n}\right)+\left(I-\alpha_{n} \mu F\right) T_{\omega} x_{n},
$$

where the sequence $\left\{\alpha_{n}\right\} \subset(0,1)$ satisfies $\lim _{n \rightarrow \infty} \alpha_{n}=0$, and $\sum_{n=0}^{\infty} \alpha_{n}=\infty$. Also $\omega \in\left(0, \frac{1}{2}\right), T_{\omega}:=(1-\omega) I+\omega I$ with two conditions on $T:$

(C1) $\|T x-q\| \leq\|x-q\|$ for any $x \in H$, and $q \in$ Fix(T); this means that $T$ is a quasi-nonexpansive mapping;

(C2) $T$ is demi-closed on $H$; that is: if $\left\{y_{k}\right\} \in H, y_{k} \rightarrow z$, and $(I-T) y_{k} \rightarrow 0$, then $z \in$ $\operatorname{Fix}(T)$.

Then, $\left\{x_{n}\right\}$ converges strongly to the $x^{*} \in F i x(T)$ which is the unique solution of the VIP:

$$
\left\langle(\mu F-\gamma f) x^{*}, x-x^{*}\right\rangle \geq 0, \quad \forall x \in \operatorname{Fix}(T) .
$$

Proof. First, we show that $\left\{x_{n}\right\}$ is bounded.

Take any $p \in \operatorname{Fix}(T)$, by Lemma 2.2 (a3), we have

$$
\begin{aligned}
& \left\|x_{n+1}-p\right\| \\
& \quad=\left\|\alpha_{n} \gamma f\left(x_{n}\right)+\left(I-\alpha_{n} \mu F\right) T_{\omega} x_{n}-p\right\| \\
& \quad=\left\|\alpha_{n} \gamma\left(f\left(x_{n}\right)-f(p)\right)+\alpha_{n}(\gamma f(p)-\mu F p)+\left(I-\alpha_{n} \mu F\right) T_{\omega} x_{n}-\left(I-\alpha_{n} \mu F\right) p\right\|(3.3 \\
& \quad \leq \alpha_{n} \gamma L\left\|x_{n}-p\right\|+\alpha_{n}\|\gamma f(p)-\mu F p\|+\left(1-\alpha_{n} \tau\right)\left\|x_{n}-p\right\| \\
& \quad \leq\left(1-\alpha_{n}(\tau-\gamma L)\right)\left\|x_{n}-p\right\|+\alpha_{n}\|\gamma f(p)-\mu F p\| .
\end{aligned}
$$

By induction, we have

$$
\left\|x_{n}-p\right\| \leq \max \left\{\left\|x_{0}-p\right\|, \frac{\|\gamma f(p)-\mu F p\|}{\tau-\gamma L}\right\}, \quad \forall n \geq 0 .
$$


Hence, $\left\{x_{n}\right\}$ is bounded, so are the $\left\{f\left(x_{n}\right)\right\}$ and $\left\{F\left(x_{n}\right)\right\}$.

From (3.1), we have

$$
x_{n+1}-x_{n}+\alpha_{n}\left(\mu F x_{n}-\gamma f\left(x_{n}\right)\right)=\left(I-\alpha_{n} \mu F\right) T_{\omega} x_{n}-\left(I-\alpha_{n} \mu F\right) x_{n} .
$$

Since $x^{*} \in F i x(T)$, from Lemma $2.2(\mathrm{a} 4)$, and together with (3.4), we obtain

$$
\begin{aligned}
& \left\langle x_{n+1}-x_{n}+\alpha_{n}\left(\mu F\left(x_{n}\right)-\gamma f\left(x_{n}\right)\right), x_{n}-x^{*}\right\rangle \\
& =\left\langle\left(I-\alpha_{n} \mu F\right) T_{\omega} x_{n}-\left(I-\alpha_{n} \mu F\right) x_{n}, x_{n}-x^{*}\right\rangle \\
& =\left(1-\alpha_{n}\right)\left\langle T_{\omega} x_{n}-x_{n}, x_{n}-x^{*}\right\rangle+\alpha_{n}\left\langle(I-\mu F) T_{\omega} x_{n}-(I-\mu F) x_{n}, x_{n}-x^{*}\right\rangle \\
& \leq-\frac{\omega}{2}\left(1-\alpha_{n}\right)\left\|x_{n}-T x_{n}\right\|^{2}+\alpha_{n}\left\|(I-\mu F) T_{\omega} x_{n}-(I-\mu F) x_{n}\right\|\left\|x_{n}-x^{*}\right\| \\
& \leq-\frac{\omega}{2}\left(1-\alpha_{n}\right)\left\|x_{n}-T x_{n}\right\|^{2}+\alpha_{n}(1-\tau)\left\|T_{\omega} x_{n}-x_{n}\right\|\left\|x_{n}-x^{*}\right\| \\
& \quad=-\frac{\omega}{2}\left(1-\alpha_{n}\right)\left\|x_{n}-T x_{n}\right\|^{2}+\omega \alpha_{n}(1-\tau)\left\|T x_{n}-x_{n}\right\|\left\|x_{n}-x^{*}\right\|,
\end{aligned}
$$

it follows from the previous inequality that

$$
\begin{aligned}
-\left\langle x_{n}-x_{n+1}, x_{n}-x^{*}\right\rangle \leq & -\alpha_{n}\left\langle(\mu F-\gamma f) x_{n}, x_{n}-x^{*}\right\rangle-\frac{\omega}{2}\left(1-\alpha_{n}\right)\left\|x_{n}-T x_{n}\right\|^{2} \\
& +\omega \alpha_{n}(1-\tau)\left\|T x_{n}-x_{n}\right\|\left\|x_{n}-x^{*}\right\| .
\end{aligned}
$$

From (iii), we obviously have

$$
\left\langle x_{n}-x_{n+1}, x_{n}-x^{*}\right\rangle=-\frac{1}{2}\left\|x_{n+1}-x^{*}\right\|^{2}+\frac{1}{2}\left\|x_{n}-x^{*}\right\|^{2}+\frac{1}{2}\left\|x_{n+1}-x_{n}\right\|^{2} .
$$

Set $\Gamma_{n}:=\frac{1}{2}\left\|x_{n}-x^{*}\right\|^{2}$, and combine (3.5) with (3.6), it follows that

$$
\begin{aligned}
\Gamma_{n+1}-\Gamma_{n}-\frac{1}{2}\left\|x_{n+1}-x_{n}\right\|^{2} \leq & -\alpha_{n}\left\langle(\mu F-\gamma f) x_{n}, x_{n}-x^{*}\right\rangle-\frac{\omega}{2}\left(1-\alpha_{n}\right)\left\|x_{n}-T x_{n}\right\|^{2} \\
& +\omega \alpha_{n}(1-\tau)\left\|T x_{n}-x_{n}\right\|\left\|x_{n}-x^{*}\right\| .
\end{aligned}
$$

Now, we calculate $\left\|x_{n}+1-x_{n}\right\|$.

From the given condition: $T_{\omega}:=(1-\omega) I+\omega T$, it is easy to deduce that $\left\|T_{\omega} x_{n}-x_{n}\right\|$ $=\omega|| x_{n}-T x_{n} \|$. Thus, it follows from (3.4) that

$$
\begin{aligned}
\left\|x_{n+1}-x_{n}\right\|^{2} & =\left\|\alpha_{n}\left(\gamma f\left(x_{n}\right)-\mu F x_{n}\right)+\left(I-\alpha_{n} \mu F\right) T_{\omega} x_{n}-\left(I-\alpha_{n} \mu F\right) x_{n}\right\|^{2} \\
& \leq 2 \alpha_{n}^{2}\left\|\gamma f\left(x_{n}\right)-\mu F x_{n}\right\|^{2}+2\left(1-\alpha_{n} \tau\right)^{2}\left\|T_{\omega} x_{n}-x_{n}\right\|^{2} \\
& =2 \alpha_{n}^{2}\left\|\gamma f\left(x_{n}\right)-\mu F x_{n}\right\|^{2}+2 \omega^{2}\left(1-\alpha_{n} \tau\right)^{2}\left\|T x_{n}-x_{n}\right\|^{2} .
\end{aligned}
$$

Then, from (3.7) and (3.8), we have

$$
\begin{aligned}
\Gamma_{n+1} & -\Gamma_{n}+\left[\frac{\omega}{2}\left(1-\alpha_{n}\right)-\omega^{2}\left(1-\alpha_{n} \tau\right)^{2}\right]\left\|x_{n}-T x_{n}\right\|^{2} \\
\leq & \alpha_{n}\left[\alpha_{n}\left\|\gamma f\left(x_{n}\right)-\mu F x_{n}\right\|^{2}-\left\langle(\mu F-\gamma f) x_{n}, x_{n}-x^{*}\right\rangle\right. \\
& \left.+\omega(1-\tau)\left\|T x_{n}-x_{n}\right\|\left\|x_{n}-x^{*}\right\|\right] .
\end{aligned}
$$

Finally, we prove $x_{n} \rightarrow x^{*}$. To this end, we consider two cases.

Case 1: Suppose that there exists $n_{0}$ such that $\left\{\Gamma_{n}\right\}_{n \geq n_{0}}$ is nonincreasing, it is equal to $\Gamma_{n+1} \leq \Gamma_{n}$ for all $n \geq n_{0}$. It follows that $\lim _{n \rightarrow \infty} \Gamma_{n}$ exists, so we conclude that

$$
\lim _{n \rightarrow \infty}\left(\Gamma_{n+1}-\Gamma_{n}\right)=0 \text {. }
$$


It follows from (3.9),(3.10) and combine with the fact that $\lim _{n \rightarrow \infty} \alpha_{n}=0$, we have $\lim _{n \rightarrow \infty}|| x_{n}-T x_{n} \|=0$. Considering (3.9) again, from (3.10), we have

$$
\begin{aligned}
& -\alpha_{n}\left[\alpha_{n}\left\|\gamma f\left(x_{n}\right)-\mu F x_{n}\right\|^{2}-\left\langle(\mu F-\gamma f) x_{n}, x_{n}-x^{*}\right\rangle\right. \\
& \left.+\omega(1-\tau)\left\|T x_{n}-x_{n}\right\|\left\|x_{n}-x^{*}\right\|\right] \\
& \quad \leq \Gamma_{n}-\Gamma_{n+1} .
\end{aligned}
$$

Then, by $\sum_{n=0}^{\infty} \alpha_{n}=\infty$, we conclude that

$$
\begin{aligned}
& \liminf _{n \rightarrow \infty}-\left[\alpha_{n}\left\|\gamma f\left(x_{n}\right)-\mu F x_{n}\right\|^{2}-\left\langle(\mu F-\gamma f) x_{n}, x_{n}-x^{*}\right\rangle\right. \\
& \left.\quad+\omega(1-\tau)\left\|T x_{n}-x_{n}\right\|\left\|x_{n}-x^{*}\right\|\right] \\
& \quad \leq 0 .
\end{aligned}
$$

Since $\left\{f\left(x_{n}\right)\right\}$ and $\left\{x_{n}\right\}$ are both bounded, as well as $\alpha_{n} \rightarrow 0$, and $\lim _{n \rightarrow \infty}|| x_{n}-T x_{n} \|=$ 0 , it follows from (3.12) that

$$
\liminf _{n \rightarrow \infty}\left\langle(\mu F-\gamma f) x_{n}, x_{n}-x^{*}\right\rangle \leq 0 .
$$

From Lemma 2.1, it is obvious that

$$
\left\langle(\mu F-\gamma f) x_{n}, x_{n}-x^{*}\right\rangle \geq\left\langle(\mu F-\gamma f) x^{*}, x_{n}-x^{*}\right\rangle+2(\mu \eta-\gamma L) \Gamma_{n} .
$$

Thus, from (3.14), and the fact that $\lim _{n \rightarrow \infty} \Gamma_{n}$ exists, we immediately obtain ********

$$
\begin{aligned}
& \liminf _{n \rightarrow \infty}\left\langle(\mu F-\gamma f) x^{*}, x_{n}-x^{*}\right\rangle+2(\mu \eta-\gamma L) \Gamma_{n} \\
& \quad=2(\mu \eta-\gamma L) \lim _{n \rightarrow \infty} \Gamma_{n}+\liminf _{n \rightarrow \infty}\left\langle(\mu F-\gamma f) x^{*}, x_{n}-x^{*}\right\rangle \leq 0,
\end{aligned}
$$

or equivalently

$$
2(\mu \eta-\gamma L) \lim _{n \rightarrow \infty} \Gamma_{n} \leq-\liminf _{n \rightarrow \infty}\left\langle(\mu F-\gamma f) x^{*}, x_{n}-x^{*}\right\rangle .
$$

Finally, by Lemma 2.6, we have

$$
2(\mu \eta-\gamma L) \lim _{n \rightarrow \infty} \Gamma_{n} \leq 0,
$$

so we conclude that $\lim _{n \rightarrow \infty} \Gamma_{n}=0$, which equivalently means that $\left\{x_{n}\right\}$ converges strongly to $x^{*}$.

Case 2: Assume that there exists a subsequence $\left\{\Gamma_{n_{j}}\right\}_{j \geq 0}$ of $\left\{\Gamma_{n}\right\}_{n} \geq 0$ such that $\Gamma_{n_{j}}<\Gamma_{n_{j}+1}$ for all $j \in \mathbb{N}$. In this case, it follows from Lemma 2.4 that there exists a subsequence $\left\{\Gamma_{\tau(n)}\right\}$ of $\left\{\Gamma_{n}\right\}$ such that $\Gamma_{\tau(n)+1}>\Gamma_{\tau(n)}$, and $\{\tau(n)\}$ is defined as in Lemma 2.4 .

Invoking (3.9) again, it follows that

$$
\begin{aligned}
\Gamma_{\tau(n)+1}-\Gamma_{\tau(n)}+\left[\frac{\omega}{2}\left(1-\alpha_{\tau(n)}\right)-\omega^{2}\left(1-\alpha_{\tau(n)} \tau\right)^{2}\right]\left\|x_{\tau(n)}-T x_{\tau(n)}\right\|^{2} \\
\leq \\
\quad \alpha_{\tau(n)}\left[\alpha_{\tau(n)}\left\|\gamma f\left(x_{\tau(n)}\right)-\mu F x_{\tau(n)}\right\|^{2}-\left\langle(\mu F-\gamma f) x_{\tau(n)}, x_{\tau(n)}-x^{*}\right\rangle\right. \\
\left.\quad+\omega(1-\tau)\left\|T x_{\tau(n)}-x_{\tau(n)}\right\|\left\|x_{\tau(n)}-x^{*}\right\|\right] .
\end{aligned}
$$


Recalling the fact that $\Gamma_{\tau(n)+1}>\Gamma_{\tau(n)}$, we have

$$
\begin{aligned}
& {\left[\frac{\omega}{2}\left(1-\alpha_{\tau(n)}\right)-\omega^{2}\left(1-\alpha_{\tau(n)} \tau\right)^{2}\right]\left\|x_{\tau(n)}-T x_{\tau(n)}\right\|^{2}} \\
& \quad \leq \alpha_{\tau(n)}\left[\alpha_{\tau(n)}\left\|\gamma f\left(x_{\tau(n)}\right)-\mu F x_{\tau(n)}\right\|^{2}-\left\langle(\mu F-\gamma f) x_{\tau(n)}, x_{\tau(n)}-x^{*}\right\rangle\right. \\
& \left.\quad+\omega(1-\tau)\left\|T x_{\tau(n)}-x_{\tau(n)}\right\|\left\|x_{\tau(n)}-x^{*}\right\|\right] .
\end{aligned}
$$

From the preceding results, we get the boundedness of $\left\{x_{n}\right\}$ and $\alpha_{n} \rightarrow 0$ which obviously lead to

$$
\lim _{n \rightarrow \infty}\left\|x_{\tau(n)}-T x_{\tau(n)}\right\|=0 .
$$

Hence, combining (3.18) with (3.19), we immediately deduce that

$$
\begin{aligned}
\left\langle(\mu F-\gamma f) x_{\tau(n)}, x_{\tau(n)}-x^{*}\right\rangle \leq & \alpha_{\tau(n)}\left\|\gamma f\left(x_{\tau(n)}\right)-\mu F x_{\tau(n)}\right\|^{2} \\
& +\omega(1-\tau)\left\|T x_{\tau(n)}-x_{\tau(n)}\right\|\left\|x_{\tau(n)}-x^{*}\right\| .
\end{aligned}
$$

Again, (3.14) and (3.20) yield

$$
\begin{aligned}
\left\langle(\mu F-\gamma f) x^{*}, x_{\tau(n)}-x^{*}\right\rangle+2(\mu \eta-\gamma L) \Gamma_{\tau(n)} \leq & \alpha_{\tau(n)}\left\|\gamma f\left(x_{\tau(n)}\right)-\mu F x_{\tau(n)}\right\|^{2} \\
& +\omega(1-\tau)\left\|T x_{\tau(n)}-x_{\tau(n)}\right\|\left\|x_{\tau(n)}-x^{*}\right\| .
\end{aligned}
$$

Recall that $\lim _{n \rightarrow \infty} \alpha_{\tau(n)}=0$, from (3.19) and (3.21), we immediately have

$$
2(\mu \eta-\gamma L) \limsup _{n \rightarrow \infty} \Gamma_{\tau(n)} \leq-\liminf _{n \rightarrow \infty}\left\langle(\mu F-\gamma f) x^{*}, x_{\tau(n)}-x^{*}\right\rangle .
$$

By Lemma 2.6, we have

$$
\liminf _{n \rightarrow \infty}\left\langle(\mu F-\gamma f) x^{*}, x_{\tau(n)}-x^{*}\right\rangle \geq 0 .
$$

Consider (3.22) again, we conclude that

$$
\limsup _{n \rightarrow \infty} \Gamma_{\tau(n)}=0,
$$

which means that $\lim _{n \rightarrow \infty} \Gamma_{\tau(n)}=0$. By Lemma 2.4, it follows that $\Gamma_{n} \leq \Gamma_{\tau(n)}$, thus, we get $\lim _{n \rightarrow \infty} \Gamma_{n}=0$, which is equivalent to $x_{n} \rightarrow x^{*}$.

Remark 3.2. Corollary 3.3 is only valid for $\omega \in\left(0, \frac{1}{2}\right)$. This is revised by Wongchan and Saejung [8].

corollary 3.3. [4] Let the sequence $\left\{x_{n}\right\}$ be generated by

$$
x_{n+1}=\alpha_{n} f\left(x_{n}\right)+\left(1-\alpha_{n}\right) T_{\omega} x_{n},
$$

where the sequence $\left\{\alpha_{n}\right\} \subset(0,1)$ satisfies $\lim _{n \rightarrow \infty} \alpha_{n}=0$, and $\sum_{n=0}^{\infty} \alpha_{n}=\infty$. Also $\omega \in\left(0, \frac{1}{2}\right)$, and $T_{\omega}:=(1-\omega) I+\omega T$ with two conditions on $T$ :

(C1) $\|T x-q\| \leq\|x-q\|$ for any $x \in H$, and $q \in$ Fix $(T)$; this means that $T$ is a quasi-nonexpansive mapping;

(C2) $T$ is demi-closed on $H$; that is: if $\left\{y_{k}\right\} \in H, y_{k} \rightarrow z$, and (I-T) $y_{k} \rightarrow 0, z \in$ Fix (T).

Then, $\left\{x_{n}\right\}$ converges strongly to the $x^{*} \in F i x(T)$ which is the unique solution of the 
$\operatorname{VIP}(3.26)$ :

$$
\left\langle(I-f) x^{*}, x-x^{*}\right\rangle \geq 0, \quad \forall x \in \operatorname{Fix}(T) .
$$

Acknowledgements

The first author was supported by the Fundamental Research Funds for the Central Universities (No. ZXH2011C002).

\section{Authors' contributions}

All authors read and approved the final manuscript.

\section{Competing interests}

The authors declare that they have no competing interests.

Received: 16 October 2011 Accepted: 25 November 2011 Published: 25 November 2011

\section{References}

1. Moudafi, A: Viscosity approximation methods for fixed-points problems. J Math Anal Appl. 241, 46-55 (2000). doi:10.1006/jmaa.1999.6615

2. Xu, HK: An iterative approach to quadratic optimizaton. J Optim Theory Appl. 116, 659-678 (2003). doi:10.1023/ A:1023073621589

3. Marino, G, Xu, HK: An general iterative method for nonexpansive mapping in Hilbert space. J Math Anal Appl. 318, 43-52 (2006). doi:10.1016/j.jmaa.2005.05.028

4. Maingé, PE: The viscosity approximation process for quasi-nonexpansive mappings in Hilbert spaces. Comput Math Appl. 59(1), 74-79 (2009)

5. Tian, M: A general iterative algorithm for nonexpansive mappings in Hilbert spaces. Nonlinear Anal. 73, 689-694 (2010). doi:10.1016/j.na.2010.03.058

6. Tian, M: A general iterative method based on the hybrid steepest descent scheme for nonexpansive mappings in Hilbert spaces. 2010 International Conference on Computational Intelligence and Software Engineering, CiSE 2010 (2010). art. no. 5677064

7. Maingé, PE: Strong convergence of projected subgradient methods for nonsmooth and nonstrictly convex minimization. Set-Valued Anal. 16(7-8), 899-912 (2008). doi:10.1007/s11228-008-0102-z

8. Wongchan, K, Saejung, S: On the strong convergence of viscosity approximation process of quasi-nonexpansive mappings in Hilbert spaces. J Abstr Appl Anal 2011, 9 (2011). Article ID 385843

doi:10.1186/1687-1812-2011-88

Cite this article as: Tian and Jin: Strong convergent result for quasi-nonexpansive mappings in Hilbert spaces.

Fixed Point Theory and Applications 2011 2011:88.

\section{Submit your manuscript to a SpringerOpen ${ }^{\circ}$} journal and benefit from:

- Convenient online submission

- Rigorous peer review

- Immediate publication on acceptance

- Open access: articles freely available online

- High visibility within the field

- Retaining the copyright to your article

Submit your next manuscript at $>$ springeropen.com 